\title{
Dark Energy density in models with Split Supersymmetry and degenerate vacua
}

\author{
C. Froggatt ${ }^{a}$, R. Nevzorov ${ }^{1} 1$, H. B. Nielsen ${ }^{c}$ \\ a School of Physics and Astronomy, University of Glasgow, Glasgow, UK \\ ${ }^{b}$ Department of Physics and Astronomy, University of Hawaii, Honolulu, USA \\ ${ }^{c}$ The Niels Bohr Institute, University of Copenhagen, Copenhagen, Denmark
}

\begin{abstract}
In $N=1$ supergravity supersymmetric (SUSY) and non-supersymmetric Minkowski vacua originating in the hidden sector can be degenerate. In the supersymmetric phase in flat Minkowski space non-perturbative supersymmetry breakdown may take place in the observable sector, inducing a non-zero and positive vacuum energy density. Assuming that such a supersymmetric phase and the phase in which we live are degenerate, we estimate the value of the cosmological constant. We argue that the observed value of the dark energy density can be reproduced in the Split-SUSY scenario of the supersymmetry breaking if the SUSY breaking scale is of order of $10^{10} \mathrm{GeV}$.
\end{abstract}

\footnotetext{
${ }^{1}$ On leave of absence from the Theory Department, ITEP, Moscow, Russia.
} 


\section{Introduction}

The tiny value of the cosmological constant (dark energy), which is responsible for the accelerated expansion of the Universe and constitutes $70 \%-73 \%$ of its energy density, is a major puzzle for modern particle physics. A fit to the recent data shows that its value is $\Lambda \sim 10^{-123} M_{P l}^{4} \sim 10^{-55} M_{Z}^{4}$ [1]. At the same time much larger contributions should come from electroweak symmetry breaking $\left(\sim 10^{-67} M_{P l}^{4}\right)$ and QCD condensates $\left(\sim 10^{-79} M_{P l}^{4}\right)$. Moreover the contribution of zero-modes is expected to push the vacuum energy density even higher up to $\sim M_{P l}^{4}$, i.e.

$$
\rho_{\Lambda} \simeq \sum_{\text {bosons }} \frac{\omega_{b}}{2}-\sum_{\text {fermions }} \frac{\omega_{f}}{2}=\int_{0}^{\Omega}\left[\sum_{b} \sqrt{|\vec{k}|^{2}+m_{b}^{2}}-\sum_{f} \sqrt{|\vec{k}|^{2}+m_{f}^{2}}\right] \frac{d^{3} \vec{k}}{2(2 \pi)^{3}} \sim-\Omega^{4}
$$

where the $m_{b}$ and $m_{f}$ are the masses of bosons and fermions while $\Omega \sim M_{P l}$. Because of the enormous cancellation needed between the contributions of different condensates to $\Lambda$, the smallness of the cosmological constant should be regarded as a fine-tuning problem.

The smallness of the cosmological constant could be related to an almost exact symmetry. An exact global supersymmetry (SUSY) ensures zero value for the energy density at the minimum of the potential of the scalar fields. However the breakdown of supersymmetry induces a huge and positive contribution to the total vacuum energy density of order $M_{S}^{4}$, where $M_{S}$ is the SUSY breaking scale. Because superpartners of quarks and leptons have not yet been observed, $M_{S}$ is expected to be higher than $100 \mathrm{GeV}$.

Since we are interested in the value of the cosmological constant, we must include gravity and thus local supersymmetry in our consideration. In supergravity (SUGRA) an enormous fine-tuning is also required to keep the cosmological constant around its observed value [2]. Our basic scenario for evaluating the tiny value of the cosmological constant implies that the SUGRA scalar potential of the hidden sector has a supersymmetric Minkowski minimum (second vacuum), in addition to the physical vacuum in which we live. In this second vacuum the supersymmetry is expected to be broken dynamically in the observable sector when the supersymmetric QCD interaction becomes non-perturbative. The physical and the second vacua are assumed to be degenerate, so that the estimated vacuum energy density in the second vacuum is then transferred to our vacuum.

The assumed degeneracy of the vacua is supposed to be justified by the so-called Multiple Point Principle (MPP). According to the MPP, Nature chooses values of coupling constants such that many phases of the underlying theory should coexist [3]. On the phase diagram of the theory it corresponds to a special point - the multiple point. The vacuum energy densities of these different phases are degenerate at the multiple point. In this paper we shall apply MPP to $(N=1)$ supergravity to give degeneracy between the two 
vacua mentioned above. This would normally require an extra fine-tuning associated with the presence of the second vacuum [4]. However, the breakdown of global symmetries, which are chosen to protect a zero value for the cosmological constant in SUGRA models, may lead to a set of degenerate vacua with broken and unbroken supersymmetry (SUSY) whose vacuum energy densities vanish in the leading approximation [5]-[6], resulting in a natural realization of the MPP conditions [3]. In our previous articles the MPP assumption was used to estimate the value of the cosmological constant in such SUGRA models inspired by degenerate vacua [4]-[7]. It is the main purpose of the present article to use the same idea for estimating the cosmological constant, but now under the assumption of a Split SUSY picture.

Instead of just postulating the MPP, a large set of approximately degenerate vacua with broken and unbroken supersymmetry can also arise from the vast landscape of string theory vacua, if the vacua with large cosmological constants are not allowed. Recently it was realized that the presence of an enormous number of long-lived metastable vacua favors high-scale breaking of supersymmetry, which motivated the introduction of the Split SUSY scenario. In this paper we attempt to estimate the value of the dark energy in the Split SUSY model, assuming the degeneracy of vacua with broken and unbroken supersymmetry (i.e. MPP). We argue that the observed value of the cosmological constant can be reproduced in the considered case, if the SUSY breaking scale is $M_{S} \sim 10^{9}-10^{10} \mathrm{GeV}$. This Split SUSY scenario predicts extremely long-lived gluinos.

The paper is organized as follows. In the next section we present $(N=1)$ SUGRA models in which the MPP conditions are fulfilled. In section 3 we discuss the Split SUSY scenario, and in section 4 we present some numerical estimates of the vacuum energy density and consider possible phenomenological implications. Our results are summarized in section 5.

\section{SUGRA models inspired by degenerate vacua}

The full $(N=1)$ SUGRA Lagrangian [8]-9] is specified in terms of an analytic gauge kinetic function $f_{a}\left(\phi_{M}\right)$ and a real gauge-invariant Kähler function $G\left(\phi_{M}, \phi_{M}^{*}\right)$, which depend on the chiral superfields $\phi_{M}$. The function $f_{a}\left(\phi_{M}\right)$ determines the gauge coupling constants $\operatorname{Re} f_{a}\left(\phi_{M}\right)=1 / g_{a}^{2}$, where the index $a$ designates different gauge groups. The Kähler function is a combination of two functions

$$
G\left(\phi_{M}, \phi_{M}^{*}\right)=K\left(\phi_{M}, \phi_{M}^{*}\right)+\ln \left|W\left(\phi_{M}\right)\right|^{2}
$$

where $K\left(\phi_{M}, \phi_{M}^{*}\right)$ is the Kähler potential while $W\left(\phi_{M}\right)$ is the superpotential of the con-

sidered SUSY model. Here we use standard supergravity mass units: $\frac{M_{P l}}{\sqrt{8 \pi}}=1$. 
The SUGRA scalar potential is given by

$$
\begin{gathered}
V\left(\phi_{M}, \phi_{M}^{*}\right)=\sum_{M, \bar{N}} e^{G}\left(G_{M} G^{M \bar{N}} G_{\bar{N}}-3\right)+\frac{1}{2} \sum_{a}\left(D^{a}\right)^{2}, \quad D^{a}=g_{a} \sum_{i, j}\left(G_{i} T_{i j}^{a} \phi_{j}\right), \\
G_{M} \equiv \frac{\partial G}{\partial \phi_{M}}, \quad G_{\bar{M}} \equiv \frac{\partial G}{\partial \phi_{M}^{*}}, \quad G_{\bar{N} M} \equiv \frac{\partial^{2} G}{\partial \phi_{N}^{*} \partial \phi_{M}}, \quad G^{M \bar{N}}=G_{\bar{N} M}^{-1},
\end{gathered}
$$

where $g_{a}$ is the gauge coupling constant associated with the generator $T^{a}$ of the gauge transformations. In order to break supersymmetry in $(N=1)$ SUGRA models, a hidden sector is introduced. It is assumed that the superfields of the hidden sector $\left(z_{i}\right)$ interact with the observable ones only by means of gravity. At the minimum of the scalar potential (3), hidden sector fields acquire vacuum expectation values (VEVs) so that at least one of their auxiliary fields

$$
F^{M}=e^{G / 2} G^{M \bar{P}} G_{\bar{P}}
$$

gets a non-vanishing VEV, giving rise to the breakdown of local SUSY and generating a non-zero gravitino mass $m_{3 / 2}=<e^{G / 2}>$.

As mentioned in section 1, the successful implementation of the MPP in $(N=1)$ supergravity requires us to assume the existence of a vacuum in which the low-energy limit of the considered theory is described by a pure supersymmetric model in flat Minkowski space. According to the MPP this vacuum and the physical one must be degenerate. Such a second vacuum is realised only if the SUGRA scalar potential has a minimum where $m_{3 / 2}=0$. The corresponding minimum is achieved when the superpotential $W$ for the hidden sector and its derivatives vanish, i.e.

$$
\begin{gathered}
W\left(z_{m}^{(2)}\right)=0 \\
\left.\frac{\partial W\left(z_{i}\right)}{\partial z_{m}}\right|_{z_{m}=z_{m}^{(2)}}=0
\end{gathered}
$$

where $z_{m}^{(2)}$ denote vacuum expectation values of the hidden sector fields in the second vacuum. In general Eq. (5) represents the extra fine-tuning associated with the presence of the supersymmetric Minkowski vacuum.

The simplest Kähler potential and superpotential that satisfies conditions (5) and (6) can be written as

$$
K\left(z, z^{*}\right)=|z|^{2}, \quad W(z)=m_{0}(z+\beta)^{2} .
$$

The hidden sector of this SUGRA model contains only one singlet superfield $z$. If the parameter $\beta=\beta_{0}=-\sqrt{3}+2 \sqrt{2}$, the SUGRA scalar potential of the considered model possesses two degenerate minima with zero energy density at the classical level. One of them is a supersymmetric Minkowski minimum that corresponds to $z^{(2)}=-\beta$. In the 
other minimum of the SUGRA scalar potential $\left(z^{(1)}=\sqrt{3}-\sqrt{2}\right)$ local supersymmetry is broken; so it can be associated with the physical vacuum. Varying the parameter $\beta$ around $\beta_{0}$ one can obtain a positive or a negative contribution from the hidden sector to the total energy density of the physical vacuum. Thus $\beta$ can be fine-tuned so that the physical and second vacua are degenerate.

The obvious drawback of the model discussed above is related with the degree of the fine-tuning which is required, in order to arrange for the presence of a supersymmetric Minkowski vacuum as well as for the degeneracy of the physical and second vacua. Nevertheless in some SUGRA models this fine-tuning can be alleviated. Let us consider the no-scale inspired SUGRA model with two hidden sector fields ( $T$ and $z$ ) and a set of chiral supermultiplets $\varphi_{\sigma}$ in the observable sector. These superfields transform differently under the imaginary translations $\left(T \rightarrow T+i \beta, z \rightarrow z, \varphi_{\sigma} \rightarrow \varphi_{\sigma}\right)$ and dilatations $\left(T \rightarrow \alpha^{2} T, z \rightarrow \alpha z, \varphi_{\sigma} \rightarrow \alpha \varphi_{\sigma}\right)$, which are subgroups of the $S U(1,1)$ group [10] [11] 2 . In order to ensure the appropriate breakdown of local supersymmetry, we assume that there is a weak breaking of the dilatation invariance of the hidden sector superpotential characterised by an hierarchically small parameter $\varkappa$. The full superpotential of the model is given by [6]:

$$
\begin{gathered}
W\left(z, \varphi_{\alpha}\right)=W_{h i d}+W_{o b s}, \\
W_{h i d}=\varkappa\left(z^{3}+\mu_{0} z^{2}+\sum_{n=4}^{\infty} c_{n} z^{n}\right), \quad W_{o b s}=\sum_{\sigma, \beta, \gamma} \frac{1}{6} Y_{\sigma \beta \gamma} \varphi_{\sigma} \varphi_{\beta} \varphi_{\gamma} .
\end{gathered}
$$

The superpotential (8) contains a bilinear mass term for the superfield $z$ and higher order terms $c_{n} z^{n}$ that spoil dilatation invariance. A term proportional to $z$ is not included. It can be forbidden by a gauge symmetry of the hidden sector, if $z$ transforms non-trivially under the corresponding gauge transformations. Here we do not allow the breakdown of dilatation invariance in the superpotential of the observable sector, in order to avoid the appearance of potentially dangerous terms which lead, for instance, to the so-called $\mu$-problem.

We also assume that the dilatation invariance is broken in the Kähler potential of the

\footnotetext{
${ }^{2}$ The Lagrangian for a no-scale SUGRA model is invariant under imaginary translations and dilatations. As a consequence the vacuum energy density goes to zero near global minima of the tree-level scalar potential which vanishes identically along some directions [8], [1] $-[12$. Thus imaginary translations and dilatations protect a zero value for the cosmological constant in supergravity. However these symmetries also preserve supersymmetry in all vacua, which has to be broken in any phenomenologically acceptable theory.
} 
observable sector, so that the full Kähler potential takes the form [6]:

$$
\begin{aligned}
K\left(\phi_{M}, \phi_{M}^{*}\right) & =-3 \ln \left[T+\bar{T}-|z|^{2}-\sum_{\alpha} \zeta_{\alpha}\left|\varphi_{\alpha}\right|^{2}\right]+ \\
& +\sum_{\alpha, \beta}\left(\frac{\eta_{\alpha \beta}}{2} \varphi_{\alpha} \varphi_{\beta}+\text { h.c. }\right)+\sum_{\beta} \xi_{\beta}\left|\varphi_{\beta}\right|^{2},
\end{aligned}
$$

where $\zeta_{\alpha}, \eta_{\alpha \beta}, \xi_{\beta}$ are some constants. In the limit when $\eta_{\alpha \beta}, \xi_{\beta}$ and $\varkappa$ go to zero, the dilatation invariance is restored, protecting supersymmetry and a zero value of the cosmological constant. It is worth noticing that we only allow the breakdown of the dilatation invariance in the Kähler potential of the observable sector, since any variations in the Kähler potential of the hidden sector may spoil the vanishing of the vacuum energy density in global minima. We restrict our consideration to the simplest set of terms that break dilatation invariance in the Kähler potential. Additional terms which are proportional to $\left|\varphi_{\alpha}\right|^{2}$ normally appear in minimal SUGRA models [13]. The other terms $\eta_{\alpha \beta} \varphi_{\alpha} \varphi_{\beta}$ introduced in the Kähler potential (9) give rise to effective $\mu$ terms after the spontaneous breakdown of local supersymmetry, solving the $\mu$ problem [14].

In the considered SUGRA model the scalar potential is positive definite

$$
\begin{gathered}
V=V_{h i d}+V_{o b s} \\
V_{h i d}=\frac{1}{3\left(T+\bar{T}-|z|^{2}\right)^{2}}\left|\frac{\partial W_{h i d}(z)}{\partial z}\right|^{2}, \\
V_{o b s}=\sum_{\alpha}\left|\frac{\partial W_{e f f}\left(y_{\beta}\right)}{\partial y_{\alpha}}+m_{\alpha} y_{\alpha}^{*}\right|^{2}+\frac{1}{2} \sum_{a}\left(D^{a}\right)^{2},
\end{gathered}
$$

so that the vacuum energy density vanishes near its global minima. In Eq. (12) $y_{\alpha}$ are canonically normalized scalar fields

$$
y_{\alpha}=\tilde{C}_{\alpha} \varphi_{\alpha}, \quad \tilde{C}_{\alpha}=\xi_{\alpha}\left(1+\frac{1}{x_{\alpha}}\right), \quad x_{\alpha}=\frac{\xi_{\alpha}<\left(T+\bar{T}-|z|^{2}\right)>}{3 \zeta_{\alpha}} .
$$

The mass parameters $m_{\alpha}$ are given by [6]

$$
m_{\alpha}=\frac{m_{3 / 2} x_{\alpha}}{1+x_{\alpha}}
$$

where $m_{3 / 2}$ denotes the gravitino mass. The effective superpotential, which describes the interactions of observable superfields at low energies, is given by

$$
\begin{gathered}
W_{e f f}=\sum_{\alpha, \beta} \frac{\mu_{\alpha \beta}}{2} y_{\alpha} y_{\beta}+\sum_{\alpha, \beta, \gamma} \frac{h_{\alpha \beta \gamma}}{6} y_{\alpha} y_{\beta} y_{\gamma}, \\
\mu_{\alpha \beta}=m_{3 / 2} \eta_{\alpha \beta}\left(\tilde{C}_{\alpha} \tilde{C}_{\beta}\right)^{-1}, \quad h_{\alpha \beta \gamma}=\frac{Y_{\alpha \beta \gamma}\left(\tilde{C}_{\alpha} \tilde{C}_{\beta} \tilde{C}_{\gamma}\right)^{-1}}{<\left(T+\bar{T}-|z|^{2}\right)^{3 / 2}>} .
\end{gathered}
$$

The form of the superpotential (8) guarantees that there is always a supersymmetric Minkowski minimum at $z=0$, because the conditions (5) and (6) are fulfilled for $z=0$ 
without any extra fine-tuning. In the simplest case when $c_{n}=0, V_{\text {hid }}$ has two minima, at $z=0$ and $z=-\frac{2 \mu_{0}}{3}$. At these points the scalar potential (11) achieves its absolute minimal value i.e. zero. In the first vacuum, where $z=-\frac{2 \mu_{0}}{3}$, local supersymmetry is broken so that the gravitino becomes massive

$$
m_{3 / 2}=\left\langle\frac{W(z)}{\left(T+\bar{T}-|z|^{2}\right)^{3 / 2}}\right\rangle=\frac{4 \kappa \mu_{0}^{3}}{27\left\langle\left(T+\bar{T}-\frac{4 \mu_{0}^{2}}{9}\right)^{3 / 2}\right\rangle} .
$$

and all scalar particles get non-zero masses $m_{\sigma} \sim \frac{m_{3 / 2} \xi_{\sigma}}{\zeta_{\sigma}}$. Assuming that $\xi_{\alpha}, \zeta_{\alpha}, \mu_{0}$ and $<T>$ are all of order unity, a supersymmetry breaking scale $M_{S} \sim 1 \mathrm{TeV}$ can only be obtained for extremely small values of $\varkappa \simeq 10^{-15}$. In the second minimum, with $z=0$, the superpotential of the hidden sector vanishes and local SUSY remains intact, so that the low-energy limit of this theory is described by a pure SUSY model in flat Minkowski space. If the high order terms $c_{n} z^{n}$ are present in Eqs. (8), the scalar potential of the hidden sector may have many degenerate vacua, with broken and unbroken supersymmetry, in which the vacuum energy density vanishes.

In the first vacuum, where the gravitino gains a non-zero mass $m_{3 / 2}$, the breakdown of electroweak (EW) symmetry can also be achieved, if the Higgs sector involves two Higgs doublets $\left(H_{u}\right.$ and $\left.H_{d}\right)$ and a singlet field $S$. The most general superpotential that describes the interactions of the Higgs superfields at low energies can be written as

$$
W_{H}=\lambda S\left(H_{u} H_{d}\right)-\frac{\sigma}{3} S^{3}-\mu\left(H_{u} H_{d}\right)-\frac{\mu^{\prime}}{2} S^{2} .
$$

We assume that the Lagrangian of the considered model is invariant under permutation symmetry $H_{u} \leftrightarrow H_{d}$ so that the soft scalar masses $m_{H_{u}}^{2}$ and $m_{H_{d}}^{2}$ are equal, i.e. $m_{H_{u}}=m_{H_{d}}=m_{H}$. Then the scalar potential (12) has a global minimum with zero vacuum energy density at which the EW symmetry is broken by the vacuum expectation values (VEVs) of the Higgs fields

$$
<S>=s=\frac{\mu-m_{H}}{\lambda}, \quad<H_{u}>=<H_{d}>= \pm \sqrt{\frac{\sigma s^{2}+\mu^{\prime} s-m_{S} s}{\lambda}},
$$

where $m_{S}$ is the soft scalar mass of the singlet field $S$. The scalar potential also has another minimum with the same energy density, where the Higgs field VEVs all vanish and in which the EW symmetry remains intact. In the supersymmetric vacuum $\mu, \mu^{\prime}$, $m_{H}$ and $m_{S}$ vanish and the Higgs fields do not acquire VEVs, so that EW symmetry does not get broken. Thus we find that there are three degenerate vacua: the supersymmetric Minkowski vacuum and two with SUSY broken in the hidden sector, one of which has unbroken EW symmetry and the other has broken EW symmetry as in the physical vacuum. As a consequence the MPP conditions are fulfilled automatically without any extra fine-tuning at the tree-level. 
It is worth emphasizing here that, even after the EW symmetry breaking, the total vacuum energy density remains zero at the minimum where SUSY is broken so that vacua with broken and unbroken supersymmetry are degenerate. Thus, in the considered case, the breakdown of dilatation invariance provides a mechanism that leads to the cancellation of the contribution to the total vacuum energy density arising from the breakdown of the EW symmetry. At the same time this breakdown of dilatation invariance also ensures the cancellation of the vacuum energy density which originates from the breakdown of local supersymmetry.

Of course, this remarkable cancellation takes place at the tree level only. The inclusion of perturbative and non-perturbative corrections to the considered SUGRA Lagrangian, which should depend on the structure of the underlying theory, are expected to spoil the degeneracy of vacua inducing a huge energy density in the vacuum where SUSY is broken. Moreover the considered SUGRA model has other serious shortcomings as well. In particular, the mechanism for the stabilization of the vacuum expectation value of the hidden sector field $T$ remains unclear. As a result the gravitino mass (see Eq. (16)) and the supersymmetry breaking scale are not fixed in the vacuum where SUSY is broken. Therefore the SUGRA model discussed above should be considered as a toy example. This model demonstrates that, in $(N=1)$ supergravity, there might be a mechanism which ensures the cancellation of different contributions to the total vacuum energy density in the physical vacuum. This mechanism may also lead to a set of degenerate vacua with broken and unbroken supersymmetry, resulting in a natural realization of the multiple point principle.

\section{Split SUSY and the Landscape}

In the present section we want to discuss the Split SUSY scenario, which we shall use in section 4 for our estimate of the cosmological constant. For this estimation it is crucial that we assume that the gauge couplings $1 / g_{a}^{2}=\operatorname{Re}_{a}(T, z)$ are the same in the phase in which we live and in the supersymmetric phase. This assumption is of course only justified if the kinetic functions $f_{a}(T, z)$ are approximately constant. Due to the mild dependence of $f_{a}(T, z)$ on $T$ and $z$ the derivatives of the gauge kinetic function tend to be small. As a result the gauginos are typically substantially lighter than the scalar particles in the considered SUSY models, i.e. $M_{a} \ll m_{\alpha}$. Such a hierarchical structure of the particle spectrum naturally appears in models with Split Supersymmetry 3 .

\footnotetext{
${ }^{3}$ It is worth noting that it seems to be rather problematic to generate a large mass hierarchy between the gauginos and sfermions within the toy SUGRA models discussed in section 2. Indeed, even in the limit, when $f_{a}(T, z)=$ const, the non-zero gaugino masses are expected to get induced [15]. Therefore,
} 
In Split SUSY one gives up on the naturalness criterion in dealing with the electroweak scale [16]-[17]. The corresponding idea has its origin in the observation that all known models of electroweak symmetry breaking, including supersymmetric ones, require an incredible amount of fine-tuning of the vacuum energy, such that the resulting cosmological constant is as small as observed. This amount of fine-tuning is much more severe than what is required to make the Higgs boson mass free of quadratic divergence. This suggests the possibility that our notions of naturalness might be misleading, and that other finetuning mechanisms may be at work in nature.

In the Split SUSY scenario supersymmetry is not used to stabilize the weak scale. This stabilization is supposed to be provided by some other fine-tuning mechanism, which anyway is needed to explain the value of the cosmological constant. Therefore, in the considered models, the SUSY breaking scale $M_{S}$ is taken to be much above $10 \mathrm{TeV}$. All scalar particles acquire masses at this high scale $M_{S}$, except for a single neutral Higgs boson, whose mass is either finely-tuned or is preserved by some other mechanism. The gauginos and Higgsinos of this theory are chosen to lie near the $\mathrm{TeV}$ scale, so as to ensure gauge coupling unification at $M_{G U T} \sim 10^{16} \mathrm{GeV}$. Indeed, if all sfermions are heavy, the pattern of grand unification is unchanged as compared with the MSSM, since heavy sfermions form complete $S U(5)$ representations [17]. Such a modification of the MSSM spectrum does not necessarily affect the mass parameters of gauginos and Higgsinos, which can be protected by a combination of an R-symmetry and Peccei Quinn symmetry, and thus they can have weak-scale masses. Hence, a TeV-scale lightest neutralino can be an appropriate cold dark matter candidate [17]-[19]. A Split SUSY spectrum naturally arises in frameworks where the mechanism of SUSY breaking preserves an R-symmetry and/or forbids Gaugino and Higgsino mass terms [16], [18], [20].

Thus the Split SUSY scenario retains the successes of the MSSM, aside from the tuning of the light Higgs mass. At the same time some flaws inherent to the MSSM elegantly disappear, when the scalar superpartners decouple. The ultra-heavy scalars, whose masses are a priori undetermined and can in principle range from hundreds of $\mathrm{TeV}$ up to $10^{13} \mathrm{GeV}$ [17], guarantee the absence of large supersymmetric flavor changing interactions and CP violation. The generic constraints from flavour and electric dipole moment data, which set $M_{S}>100-1000 \mathrm{TeV}$, are easily satisfied within this class of models. The dimension-five operators which mediate proton decay are also suppressed, delaying proton decay which now occurs via dimension-six operators. The increase in the predicted proton lifetime is also in better agreement with data [21].

Nevertheless, since the sfermions are ultra-heavy, the large quadratic corrections to the mass of the Higgs are not cancelled in the manner present in weak-scale supersymmetry. in our case an additional fine-tuning is probably required to ensure that $\frac{M_{a}}{m_{\alpha}} \ll 10^{-2}$. 
As a consequence, in Split SUSY the Higgs sector remains extremely fine-tuned, often with the understanding that this fine-tuning could be resolved by some anthropic-like selection effects [22]. In other words the solution to both the hierarchy and cosmological constant problems might not involve natural cancellations, but follow from a completely different reasoning, such as the idea that galaxy and star formation, chemistry and biology, are simply impossible without these scales having the values found in our Universe [22]-[23]. In this case SUSY may have nothing to do with the naturalness problem, although it may be a necessary ingredient in a fundamental theory of nature such as String Theory.

It has been argued that String Theory can have an enormous number of long-lived metastable vacua [24]-[28]. The space of such string theory vacua is called the "landscape". The number of discrete vacua in String Theory is measured not in the millions or billions but in googles $\left(\sim 10^{100}\right)$ [25]-[28]. Recent developments in String Theory applied a statistical approach to the large multitude of universes, corresponding to the "landscape" of vacua present in the theory [26]-[27]. These investigations indicate that among the vast number of metastable vacua, there can be a small subset exhibiting low scale SUSY breaking. However the fine-tuning required to achieve a small cosmological constant implies the need for a much larger number of such vacua. Remarkably, the total number of vacua in String Theory can be large enough to fine-tune both the cosmological constant and the Higgs mass, favoring a high-scale breaking of supersymmetry [27]-[28]. It is thus statistically feasible in String Theory for us to live in a universe fine-tuned in the way we find it, thereby having both a small cosmological constant and the electroweak scale stabilized in the $100 \mathrm{GeV}$ range. In the vast landscape of possible string theory vacua, we may find ourselves in the observed ground state simply because of a cosmic selection rule, i.e. the anthropic principle [22].

The idea of the multiple point principle and the landscape paradigm have at least two things in common. Both approaches imply the existence of a large number of vacua with broken and unbroken supersymmetry. The landscape paradigm and MPP also imply that the parameters of the theory, which leads to the Standard Model (SM) at low energies, can be extremely fine-tuned so as to ensure a tiny vacuum energy density and a large hierarchy between $M_{P l}$ and the electroweak scale. Moreover the MPP assumption may originate from the landscape of string theory vacua, if all vacua with a large cosmological constant are forbidden for some reason so that all the allowed string vacua, with broken and unbroken supersymmetry, are approximately degenerate. If this is the case, then the breaking of supersymmetry at high scales is probably still favored. Therefore our attempt to estimate the value of the cosmological constant within the Split SUSY scenario assuming the degeneracy of vacua with broken and unbroken supersymmetry might not be so inconsistent with the string landscape picture. 
If one assumes that for some reason numerically large cosmological constants get forbidden, the landscape model leads to what we might call a landscape and forbiddenness based MPP. In fact only a narrow band of values around zero cosmological constant would then be allowed and the surviving vacua would obey MPP to the accuracy of the width $w$ of this remaining band. However, this landscape and forbiddenness based MPP would never be sufficiently accurate to become relevant for the main point of the present article, according to which MPP "transfers" the cosmological constant $\Lambda_{2}$ of the supersymmetric "second vacuum" to the physical vacuum.

\section{Cosmological constant in Split SUSY scenario}

In section 2 we argued that the supersymmetric Minkowski and physical vacua can be degenerate in SUGRA models. We now try to estimate the value of the cosmological constant in such models. Thus we assume that the MPP is realized in Nature, leading to the formation of a set of degenerate vacua with broken and unbroken local supersymmetry. In the SUGRA models considered the low energy limit of the theory in the vacuum with unbroken local supersymmetry is described by a SUSY model in flat Minkowski space. So the vacuum energy density of the corresponding supersymmetric states vanishes. Because all vacua in the MPP inspired SUGRA models are degenerate, the cosmological constant problem is thereby solved to first approximation by assumption.

However the value of the cosmological constant may differ from zero in the considered models. This occurs if non-perturbative effects in the "observable sector" of the supersymmetric phase give rise to the breakdown of SUSY at low energies. Then the MPP assumption implies that the physical phase, in which local supersymmetry is broken in the hidden sector, has the same energy density as a second phase, where supersymmetry breakdown takes place in the observable sector.

The non-perturbative effects in the simplest SUSY models, like the minimal supersymmetric standard model (MSSM), are extremely weak. Our strategy is to estimate these effects in the vacuum with unbroken local supersymmetry (the second vacuum) and thereby estimate the energy density in this phase. This value of the cosmological constant can then be interpreted as the physical value in our phase, by virtue of the MPP.

If supersymmetry breaking takes place in the second vacuum, it is caused by the strong interactions. We assume the gauge couplings at high energies are identical in both vacua. Consequently the renormalization group running of the gauge couplings down to

the scale $M_{S}$, corresponding to the SUSY breaking scale in the physical vacuum, are also 
the same in both vacua 4 . Below the scale $M_{S}$ all squarks and sleptons in the physical vacuum decouple and the corresponding beta functions change. At the $\mathrm{TeV}$ scale, the beta functions in the physical vacuum change once again because of the gradual decoupling of the gluino, neutralino and chargino. Using the value of $\alpha_{3}^{(1)}\left(M_{Z}\right) \approx 0.1184 \pm 0.0007$ and the matching condition $\alpha_{3}^{(2)}\left(M_{S}\right)=\alpha_{3}^{(1)}\left(M_{S}\right)$, one finds the value of the strong coupling in the second vacuum:

$$
\frac{1}{\alpha_{3}^{(2)}\left(M_{S}\right)}=\frac{1}{\alpha_{3}^{(1)}\left(M_{Z}\right)}-\frac{\tilde{b}_{3}}{4 \pi} \ln \frac{M_{g}^{2}}{M_{Z}^{2}}-\frac{b_{3}^{\prime}}{4 \pi} \ln \frac{M_{S}^{2}}{M_{g}^{2}} .
$$

In Eq.(19) $\alpha_{3}^{(1)}$ and $\alpha_{3}^{(2)}$ are the values of the strong gauge couplings in the physical and second vacua, $M_{g}$ is the mass of the gluino, while $\tilde{b}_{3}=-7$ and $b_{3}^{\prime}=-5$ are the oneloop beta functions of the strong gauge coupling in the SM and Split SUSY scenario respectively.

The particles of the MSSM are, of course, all massless in the second supersymmetric phase, where the EW symmetry is unbroken. So, in the second vacuum, the renormalization group $(\mathrm{RG})$ flow of the strong gauge coupling is determined by the corresponding MSSM beta function, which exhibits asymptotically free behaviour $\left(b_{3}=-3\right)$. As a consequence $\alpha_{3}^{(2)}(Q)$ increases in the infrared region. Moreover the top quark is massless in the second vacuum and the top quark Yukawa coupling grows in the infrared with the increasing of $\alpha_{3}^{(2)}(Q)$. At the scale

$$
\Lambda_{S Q C D}=M_{S} \exp \left[\frac{2 \pi}{b_{3} \alpha_{3}^{(2)}\left(M_{S}\right)}\right],
$$

where the supersymmetric QCD interactions become strong in the second vacuum, the top quark Yukawa coupling is of the same order of magnitude as the strong gauge coupling. The large Yukawa coupling of the top quark may result in the formation of a quark condensate 5 that breaks supersymmetry, inducing a non-zero positive value for the cosmological constant

$$
\Lambda \simeq \Lambda_{S Q C D}^{4}
$$

In Fig. 1 the dependence of $\Lambda_{S Q C D}$ for the second vacuum on the SUSY breaking scale $M_{S}$ in the physical vacuum is examined. In our numerical analysis we set $M_{g}=500 \mathrm{GeV}$. Because $b_{3}>b_{3}^{\prime}$ the QCD gauge coupling below $M_{S}$ is larger in the physical vacuum than in the second vacuum. Therefore the value of $\Lambda_{S Q C D}$ is much lower than the QCD scale in the Standard Model and diminishes with increasing $M_{S}$. When $M_{S}$ is of the order of

\footnotetext{
${ }_{4}$ The gauge couplings obey the renormalization group equations $\frac{d \log \alpha_{i}(Q)}{d \log Q^{2}}=\frac{b_{i} \alpha_{i}(Q)}{4 \pi}$, where $\alpha_{i}(Q)=g_{i}^{2}(Q) /(4 \pi)$.

${ }^{5}$ Two of us have speculated [29] that such a new phase might even exist in the SM with a condensate of a 6 top and 6 antitop quark bound state.
} 
$1 \mathrm{TeV}$, we obtain $\Lambda_{S Q C D}=10^{-26} M_{P l} \simeq 100 \mathrm{eV}$. This leads to a vacuum energy density $\left(\Lambda \simeq 10^{-104} M_{P l}^{4}\right)$ which is much smaller than the electroweak scale contribution in our vacuum $v^{4} \simeq 10^{-62} M_{P l}$. From Fig. 1 one can see that the measured value of the cosmological constant is reproduced when $M_{S} \sim 10^{10} \mathrm{GeV}$. The value of the SUSY breaking scale, which leads to a reasonable agreement with the observed vacuum energy density, depends on $\alpha_{3}\left(M_{Z}\right)$ and the gluino mass. As one can see from Fig. 1, the dependence of $M_{S}$ on $\alpha_{3}\left(M_{Z}\right)$ is rather weak. With increasing gluino mass the value of the SUSY breaking scale, which results in an appropriate value of the cosmological constant, decreases. The results of our numerical analysis indicate that, for $\alpha_{3}\left(M_{Z}\right)=0.116-0.121$ and $M_{g}=500-2500 \mathrm{GeV}$, the value of $M_{S}$ varies from $2 \cdot 10^{9} \mathrm{GeV}$ up to $3 \cdot 10^{10} \mathrm{GeV}$.

The obtained prediction for the supersymmetry breaking scale can be tested. A striking feature of the Split SUSY model is the extremely long lifetime of the gluino. In the considered case, the gluino decays through a virtual squark to a quark antiquark pair and a neutralino $\tilde{g} \rightarrow q \bar{q}+\chi_{1}^{0}$. The large mass of the squarks then implies a long lifetime for the gluino. This lifetime is given approximately by [30]-[31]

$$
\tau \sim 8\left(\frac{M_{S}}{10^{9} \mathrm{GeV}}\right)^{4}\left(\frac{1 \mathrm{TeV}}{M_{g}}\right)^{5} s .
$$

When $M_{S} \gtrsim 10^{13} \mathrm{GeV}$ the gluino lifetime becomes larger than the age of the Universe. So long-lived gluinos would have been copiously produced during the very early epochs of the Big Bang. They would have survived annihilation and would subsequently have been confined in nuclear isotopes, which should be present in terrestrial matter [32]. Because the presence of such stable relics are ruled out by different experiments (for $M_{g} \lesssim 10 \mathrm{TeV}$ ) [33], the supersymmetry breaking scale in the Split SUSY scenario should not exceed $10^{13} \mathrm{GeV}$ [17]. If, as is predicted, the SUSY breaking scale varies from $2 \cdot 10^{9} \mathrm{GeV}\left(M_{g}=2500 \mathrm{GeV}\right)$ to $3 \cdot 10^{10} \mathrm{GeV}\left(M_{g}=500 \mathrm{GeV}\right)$, then the gluino lifetime changes from 1 sec. to $2 \cdot 10^{8}$ sec. (1000 years). Thus the measurement of the gluino lifetime will allow an estimate to be made of the value of $M_{S}$ in the Split SUSY model. The experimental signatures of longlived gluinos at colliders and in cosmic rays within the Split Supersymmetry scenario were explored in [31], [34].

The observed value of the cosmological constant can also be reproduced for a much lower supersymmetry breaking scale, if the MSSM particle content is supplemented by an additional pair of $5+\overline{5}$ supermultiplets. The extra bosons and fermions would not affect gauge coupling unification, because they form complete representations of $S U(5)$ (see for example [35]). In the physical vacuum new bosonic states gain masses around the supersymmetry breaking scale, while their fermionic partners survive to low energies. In our numerical studies we assume that the masses of any new quarks are of the order of the gluino mass, i.e. we set $M_{q} \simeq M_{g} \simeq 500 \mathrm{GeV}$. In the supersymmetric phase the 
new bosons and fermions remain massless. The extra fermionic states do not much affect the RG flow of gauge couplings in the Split SUSY scenario. For example, the one-loop beta function that determines the running of the strong gauge coupling from the SUSY breaking scale down to the $\mathrm{TeV}$ scale changes from -5 to $-13 / 3$. At the same time, the extra $5+\overline{5}$ supermultiplets give a considerable contribution to $b_{3}$ in the supersymmetric phase. The corresponding one-loop beta function becomes $b_{3}=-2$. As a result $\alpha_{3}(Q)$ and $\Lambda_{S Q C D}$ decrease. In this case the observed value of the cosmological constant can be reproduced even for $M_{S} \simeq 1 \mathrm{TeV}$ (see Fig. 1). However the Split SUSY scenario has the advantage of avoiding the need for any new particles beyond those of the MSSM, provided that $M_{S} \simeq 10^{10} \mathrm{GeV}$.

\section{Conclusions}

In the present article we have tried to estimate the value of the cosmological constant in the Split Supersymmetry model, assuming the existence of a set of degenerate vacua with broken and unbroken SUSY. Using a sufficiently accurate principle of these vacua being degenerate (MPP), a small cosmological constant is calculated in one of the vacua and transferred to the other ones, especially to the physical one in which we live. The idea is that we can calculate the cosmological constant in the supersymmetric Minkowski (second) vacuum, having unbroken local supersymmetry up to non-perturbative corrections coming from the supersymmetric QCD scale in that vacuum. With reasonable Split SUSY parameters - a SUSY breaking scale of $M_{S} \sim 10^{10} \mathrm{GeV}$ - we obtain the observed value of the cosmological constant.

We have argued that a set of such degenerate vacua can appear in $N=1$ supergravity models. In general the presence of degenerate vacua in these models requires an extra fine-tuning. Moreover an extra fine-tuning is normally needed to ensure the existence of the vacuum in which the low-energy limit of the considered theory is described by a pure supersymmetric model in flat Minkowski space. The fine-tuning mentioned above can be alleviated within the no-scale inspired SUGRA models in which the global symmetries, that protect a zero value for the cosmological constant in all vacua and preserve local supersymmetry, can be broken so that the MPP conditions are fulfilled automatically without any extra fine-tuning at the tree-level. In these models there can be a vacuum with softly broken supersymmetry and spontaneously broken EW symmetry in the observable sector just like in the physical vacuum in which we live. We demonstrated that

the contributions to the vacuum energy density, which originate from the breakdown of supersymmetry and EW symmetry breaking in the physical vacuum, might get cancelled at the tree level in these models. 


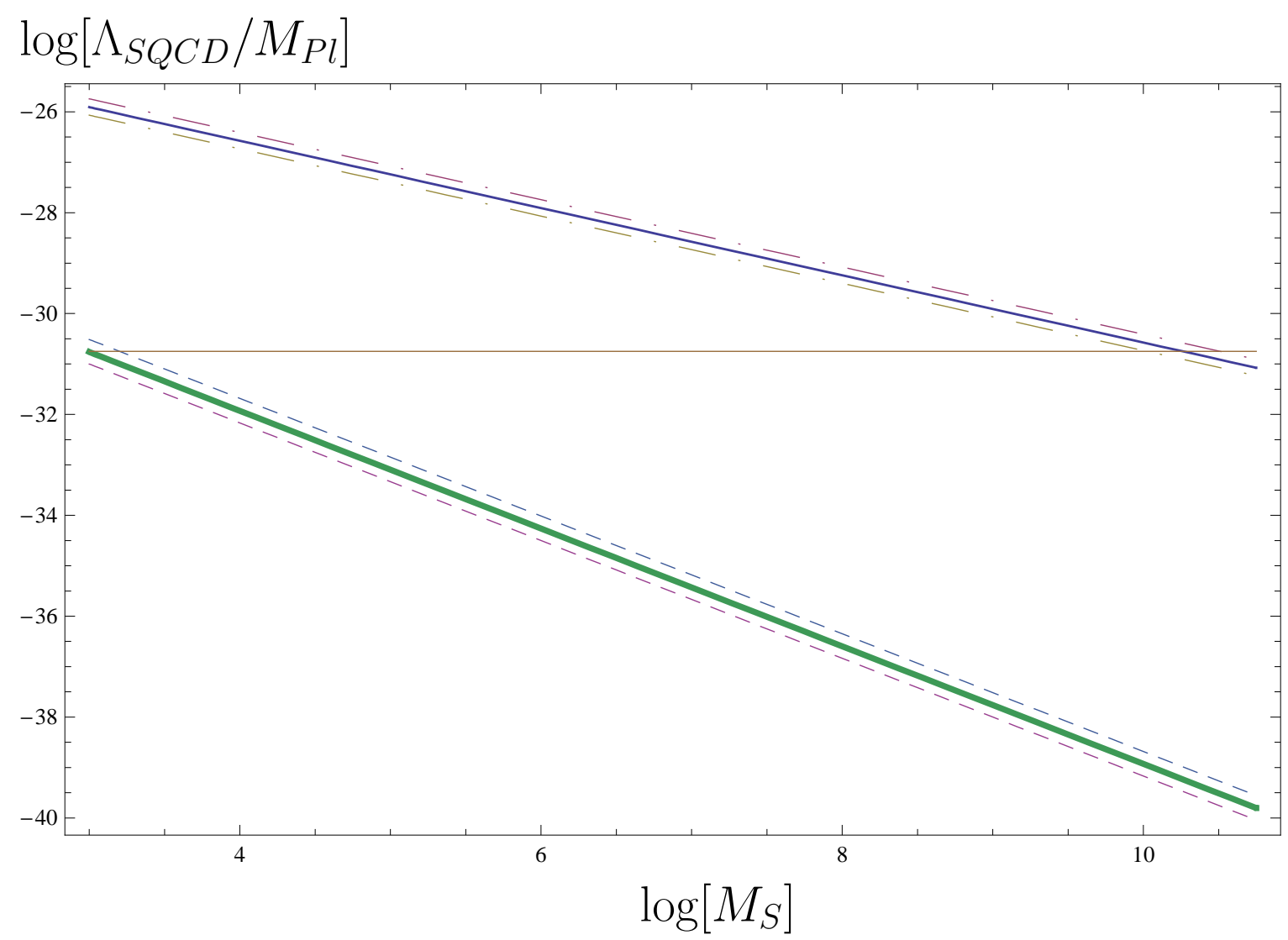

Figure 1: The value of $\log \left[\Lambda_{S Q C D} / M_{P l}\right]$ versus $\log M_{S}$ for $M_{q}=M_{g}=500 \mathrm{GeV}$. The thin and thick solid lines correspond to the Split SUSY scenarios with the pure MSSM particle content and the MSSM particle content supplemented by an additional pair of $5+\overline{5}$ multiplets respectively. The dashed and dash-dotted lines represent the uncertainty in $\alpha_{3}\left(M_{Z}\right)$. The thin and thick solid lines are obtained for $\alpha_{3}\left(M_{Z}\right)=0.1184$, the upper (lower) dashed and dash-dotted lines correspond to $\alpha_{3}\left(M_{Z}\right)=0.116\left(\alpha_{3}\left(M_{Z}\right)=0.121\right)$. The horizontal line represents the observed value of $\Lambda^{1 / 4}$. The SUSY breaking scale $M_{S}$ is measured in $\mathrm{GeV}$. 
However the inclusion of perturbative and non-perturbative corrections would spoil the degeneracy of vacua. As a consequence an enormous fine tuning is required, in general, to keep the vacuum energy densities in the vacua with broken and unbroken supersymmetry to be the same. Nevertheless for our estimation of the dark energy density we adopt such a fine-tuning assumption. In other words we assume that there might be a mechanism (approximate global symmetry, MPP or something else) in Nature that guarantees the degeneracy of the vacua mentioned above. Moreover we restrict our consideration to the simplest SUGRA models in which $f_{a}(T, z) \approx$ const. In this limit the gauginos are much lighter than squarks and sleptons, which is a characteristic feature of the Split SUSY type spectrum.

The landscape of the metastable string theory vacua favors high-scale SUSY breaking which could lead to Split SUSY. The enormous number of vacua in String Theory can also lead to a large set of approximately degenerate vacua, with broken and unbroken supersymmetry and tiny values of the vacuum energy densities, if the vacua with large cosmological constants are forbidden. Thus the Split SUSY scenario, landscape paradigm and the multiple point principle might be different parts of the same picture. This landscape based MPP is however not sufficiently accurate for the main calculation in this paper.

The main calculation in the present article concerns two of the several vacua degenerate according to the MPP assumption. The first one is the physical vacuum in which we live and in which we assume the physics is described by the Split SUSY scenario. The second vacuum is one with unbroken supersymmetry to first approximation. At high energy the physics is the same in both these vacua, so that especially the running gauge couplings are equal above the SUSY breaking scale $M_{S}$ in the physical vacuum. Also, in the second vacuum the QCD $\left(S U(3)_{C}\right)$ gauge coupling and the top quark Yukawa coupling become large towards the infrared. At the scale $\Lambda_{S Q C D}$ a top quark condensate may get formed, giving rise to the breakdown of SUSY in the supersymmetric phase and resulting in a nonzero and positive vacuum energy density. The corresponding energy density is determined by the value of the $S U(3)_{C}$ gauge coupling in the physical vacuum at high energies. In order to calculate this energy density from the experimentally known QCD coupling, the only unknown parameter is the SUSY breaking scale $M_{S}$ in the physical vacuum. For any value of $M_{S}$ the calculated vacuum energy density in the supersymmetric phase is rather small $\left(\lesssim 10^{-100} M_{P l}^{4}\right)$. The measured value of the cosmological constant can be reproduced when $M_{S} \sim 10^{9}-10^{10} \mathrm{GeV}$, which leads to extremely long-lived gluinos. The gluino lifetime varies from 1 sec. to $2 \cdot 10^{8}$ sec. This prediction can be tested if such long-lived gluinos are observed in future experiments. Previously [6] we showed that a reasonable value for the dark energy density can be obtained even for $M_{S} \sim 1 \mathrm{TeV}$ if the 
MSSM particle content is supplemented by an extra pair of $5+\overline{5}$ supermultiplets. The scenario with extra $5+\overline{5}$ multiplets of matter and the supersymmetry breaking scale in the $\mathrm{TeV}$ range can also be tested at the LHC in the near future. But with the Split SUSY scenario we avoid these ad hoc $5+\overline{5}$ particles.

\section{Acknowledgements}

R.N. would like to thank M. Drees, K. R. Dienes, D. Gorbunov, D. R. T. Jones, S. F. King, J. P. Kumar, S. Pakvasa, D. G. Sutherland, X. R. Tata, B. Thomas and M. I. Vysotsky for fruitful discussions. The work of R.N. was supported by the U.S. Department of Energy under Contract DE-FG02-04ER41291. Also C.D.F. would like to acknowledge support from STFC in UK. 


\section{References}

[1] A.G. Riess et al., Astron. J. 116, 1009 (1998);

S. Perlmutter et al., Astrophys. J. 517, 565 (1999);

C. Bennett et al., Astrophys. J. Suppl. 148, 1 (2003);

D. Spergel et al., Astrophys. J. Suppl. 148, 175 (2003).

[2] J. Polonyi, Budapest preprint KFKI-1977 93 (1977).

[3] D.L. Bennett and H.B. Nielsen, Int. J. Mod. Phys. A 9, 5155 (1994);

D.L. Bennett, C.D. Froggatt and H.B. Nielsen, in Proceedings of the 27th International Conference on High energy Physics, Glasgow, Scotland, 1994, p. 557; Perspectives in Particle Physics '94, World Scientific, 1995, p. 255, ed. D. Klabučar, I. Picek and D. Tadić arXiv:hep-ph/9504294];

H. B. Nielsen and M. Ninomiya, arXiv:hep-th/0701018;

H. B. Nielsen, Int. J. Mod. Phys. E 20, 2049 (2011) [arXiv:1103.3812 [hep-ph]].

[4] C. Froggatt, L. Laperashvili, R. Nevzorov and H. B. Nielsen, Phys. Atom. Nucl. 67 (2004) 582 [Yad. Fiz. 67 (2004) 601] [arXiv:hep-ph/0310127].

[5] C. Froggatt, L. Laperashvili, R. Nevzorov and H. B. Nielsen, in Bled Workshops in Physics (ISSN: 1580-4992), Vol. 5, No. 2, DFMA - zaloznistvo, Ljubljana, 2004, p. 17 arXiv:hep-ph/0411273.

[6] C. Froggatt, R. Nevzorov and H. B. Nielsen, Nucl. Phys. B 743 (2006) 133 arXiv:hep-ph/0511259.

[7] C. D. Froggatt, R. Nevzorov and H. B. Nielsen, J. Phys. Conf. Ser. 110 (2008) 072012 arXiv:0708.2907 [hep-ph]]; C. D. Froggatt, R. Nevzorov and H. B. Nielsen, arXiv:0810.0524 [hep-th]; C. D. Froggatt, R. Nevzorov and H. B. Nielsen, AIP Conf. Proc. 1200 (2010) 1093 [arXiv:0909.4703 [hep-ph]].

[8] A.B. Lahanas and D.V. Nanopoulos, Phys. Rep. 1451 (1987).

[9] H.P. Nilles, Phys. Rep. 110, 1 (1984).

[10] J. Ellis, M.K. Gaillard, M. Günaydin and B. Zumino, Nucl. Phys. B 224, 427 (1983).

[11] J. Ellis, C. Kounnas and D.V. Nanopoulos, Nucl. Phys. B 241, 406 (1984).

[12] E. Cremmer, S. Ferrara, C. Kounnas and D.V. Nanopoulos, Phys. Lett. B 133, 61 (1983). 
[13] R. Barbieri, S. Ferrara and C. Savoy, Phys. Lett. B 119, 343 (1982); A.H.Chamseddine, R.Arnowitt, P.Nath, Phys.Rev.Lett. 49, 970 (1982); H.P.Nilles, M.Srednicki, D.Wyler, Phys.Lett. B 120, 345 (1983).

[14] G.F. Giudice and A. Masiero, Phys. Lett. B 206, 480 (1988);

J.A. Casas and C. Muñoz, Phys. Lett. B 306, 288 (1993).

[15] L. Randall, R. Sundrum, Nucl. Phys. B 557 (1999) 79 hep-th/9810155; G. F. Giudice, M. A. Luty, H. Murayama, R. Rattazzi, JHEP 9812 (1998) 027 hep-ph/9810442; M. Dine, N. Seiberg, JHEP 0703 (2007) 040 hep-th/0701023.

[16] N. Arkani-Hamed, S. Dimopoulos, JHEP 0506 (2005) 073 arXiv:hep-th/0405159].

[17] G. F. Giudice, A. Romanino, Nucl. Phys. B 699 (2004) 65 [Erratum-ibid. B 706 (2005) 65] arXiv:hep-ph/0406088.

[18] N. Arkani-Hamed, S. Dimopoulos, G. F. Giudice and A. Romanino, Nucl. Phys. B 709 (2005) 3 arXiv:hep-ph/0409232.

[19] A. Pierce, Phys. Rev. D 70 (2004) 075006 arXiv:hep-ph/0406144; A. Masiero, S. Profumo and P. Ullio, Nucl. Phys. B 712 (2005) 86 arXiv:hep-ph/0412058.

[20] I. Antoniadis and S. Dimopoulos, Nucl. Phys. B $715 \quad(2005) \quad 120$ arXiv:hep-th/0411032; B. Kors and P. Nath, Nucl. Phys. B 711 (2005) 112 arXiv:hep-th/0411201; K. S. Babu, T. Enkhbat and B. Mukhopadhyaya, Nucl. Phys. B 720 (2005) 47 arXiv:hep-ph/0501079.

[21] H. Murayama and A. Pierce, Phys. Rev. D 65 (2002) 055009 arXiv:hep-ph/0108104.

[22] S. Weinberg, Phys. Rev. Lett. 59 (1987) 2607.

[23] V. Agrawal, S. M. Barr, J. F. Donoghue and D. Seckel, Phys. Rev. D 57 (1998) 5480 arXiv:hep-ph/9707380; C. J. Hogan, Rev. Mod. Phys. 72 (2000) 1149 arXiv:astro-ph/9909295; M. J. Rees, arXiv:astro-ph/0401424.

[24] S. Kachru, R. Kallosh, A. D. Linde and S. P. Trivedi, Phys. Rev. D 68 (2003) 046005 arXiv:hep-th/0301240; L. Susskind, arXiv:hep-th/0302219; T. Banks, M. Dine and E. Gorbatov, JHEP 0408 (2004) 058 arXiv:hep-th/0309170]; M. Dine, E. Gorbatov, S. D. Thomas, JHEP 0808 (2008) 098 [arXiv:hep-th/0407043].

[25] R. Bousso and J. Polchinski, JHEP 0006 (2000) 006 arXiv:hep-th/0004134. 
[26] M. R. Douglas, JHEP 0305 (2003) 046 arXiv:hep-th/0303194; S. Ashok and M. R. Douglas, JHEP 0401 (2004) 060 arXiv:hep-th/0307049; A. Giryavets, S. Kachru and P. K. Tripathy, JHEP 0408 (2004) 002 arXiv:hep-th/0404243; J. P. Conlon and F. Quevedo, JHEP 0410 (2004) 039 arXiv:hep-th/0409215.

[27] F. Denef and M. R. Douglas, JHEP 0405 (2004) 072 arXiv:hep-th/0404116; M. R. Douglas, Comptes Rendus Physique 5 (2004) 965 arXiv:hep-th/0409207.

[28] L. Susskind, arXiv:hep-th/0405189.

[29] C. D. Froggatt and H. B. Nielsen, Surv. High Energy Phys. 18 (2003) 55 arXiv:hep-ph/0308144]; C. D. Froggatt, L. V. Laperashvili and H. B. Nielsen, Int. J. Mod. Phys. A20 (2005) 1268 arXiv:hep-ph0406110; C. D. Froggatt and H. B. Nielsen, Phys. Rev. Lett. 95 (2005) 231301 astro-ph/0508513]; C. D. Froggatt and H. B. Nielsen, arXiv:0810.0475 [hep-ph]]; C. D. Froggatt and H. B. Nielsen, Phys. Rev. D80 (2009) 034033 [arXiv:0810.0475 [hep-ph]].

[30] S. Dawson, E. Eichten and C. Quigg, Phys. Rev. D 31 (1985) 1581.

[31] J. L. Hewett, B. Lillie, M. Masip and T. G. Rizzo, JHEP 0409 (2004) 070 arXiv:hep-ph/0408248.

[32] S. Wolfram, Phys. Lett. B 82 (1979) 65; C. B. Dover, T. K. Gaisser, G. Steigman, Phys. Rev. Lett. 42 (1979) 1117.

[33] J. Rich, M. Spiro, J. Lloyd-Owen, Phys. Rept. 151 (1987) 239; P. F. Smith, Contemp. Phys. 29 (1988) 159; T. K. Hemmick et al. , Phys. Rev. D 41 (1990) 2074.

[34] A. Arvanitaki, C. Davis, P. W. Graham, A. Pierce and J. G. Wacker, Phys. Rev. D 72 (2005) 075011 arXiv:hep-ph/0504210]; L. Anchordoqui, H. Goldberg and C. Nunez, Phys. Rev. D 71 (2005) 065014 arXiv:hep-ph/0408284; W. Kilian, T. Plehn, P. Richardson and E. Schmidt, Eur. Phys. J. C 39 (2005) 229 arXiv:hep-ph/0408088.

[35] R. Hempfling, Phys. Lett. B 351 (1995) 206. 